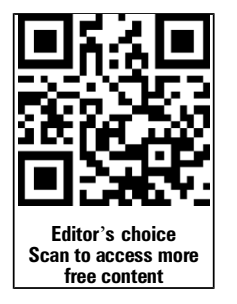

\title{
Divergent CSF $\tau$ alterations in two common tauopathies: Alzheimer's disease and progressive supranuclear palsy
}

\author{
Dana Wagshal, ${ }^{1}$ Sethu Sankaranarayanan, ${ }^{2}$ Valerie Guss, ${ }^{2}$ Tracey Hall, ${ }^{2}$ Flora Berisha, ${ }^{3}$ \\ Iryna Lobach, ${ }^{1}$ Anna Karydas, ${ }^{1}$ Lisa Voltarelli, ${ }^{1}$ Carole Scherling, ${ }_{1}^{1}$ Hilary Heuer, ${ }^{1}$ \\ Maria Carmela Tartaglia, ${ }^{1,4}$ Zachary Miller, ${ }^{1}$ Giovanni Coppola, ${ }^{5}$ Michael Ahlijanian, ${ }^{2}$ \\ Holly Soares, ${ }^{2}$ Joel H Kramer, ${ }^{1}$ Gil D Rabinovici, ${ }^{1}$ Howard J Rosen, ${ }^{1}$ Bruce L Miller, ${ }^{1}$ \\ Jere Meredith, ${ }^{2}$ Adam L Boxer ${ }^{1}$
}

'Department of Neurology, Memory and Aging Center, University of California, San Francisco, San Francisco, California, USA

${ }^{2}$ Bristol-Myers Squibb,

Wallingford, Connecticut, USA ${ }^{3}$ Kyowa Hakko Kirin Pharma, Inc., Princeton, New Jersey, USA

${ }^{4}$ Tanz Center for Research in Neurodegenerative disease, University of Toronto, Toronto, Canada

${ }^{5}$ Department of Psychiatry, Semel Institute, University of California, Los Angeles, Los Angeles, California, USA

\section{Correspondence to} Dr Adam L Boxer, Sandler Neurosciences Center, Suite 190, University of California, San Francisco; Box 1207, San Francisco, CA 94158, USA; aboxer@memory.ucsf.edu

Received 3 March 2014 Revised 24 April 2014 Accepted 17 May 2014 Published Online First 4 June 2014

\section{SLinked}

- http://dx.doi.org/10.1136/ jnnp-2014-308567

\section{CrossMark}

To cite: Wagshal $D$, Sankaranarayanan S, Guss V, et al. I Neurol Neurosurg Psychiatry 2015;86: 244-250.

\begin{abstract}
Background Elevated CSF $\tau$ is considered a biomarker of neuronal injury in newly developed Alzheimer's disease (AD) and mild cognitive impairment (MCI) criteria. However, previous studies have failed to detect alterations of $\tau$ species in other primary tauopathies. We assessed CSF $\tau$ protein abnormalities in $A D$, a tauopathy with prominent $A \beta$ pathology, and progressive supranuclear palsy (PSP), a primary tauopathy characterised by deposition of four microtubule-binding repeat (4R) $\tau$ with minimal $A \beta$ pathology.
\end{abstract}

Methods 26 normal control (NC), 37 AD, and 24 patients with PSP participated in the study. AD and PSP were matched for severity using the clinical dementia rating sum of boxes (CDR-sb) scores. The INNO BIA AlzBio3 multiplex immunoassay was used to measure CSF A $\beta$, total $\tau$, and ptau181. Additional, novel ELISAs targeting different $\mathrm{N}$-terminal and central $\tau$ epitopes were developed to examine CSF $\tau$ components and to investigate interactions between diagnostic group, demographics and genetic variables.

Results PSP had lower CSF N-terminal and C-terminal $\tau$ concentrations than $N C$ and $A D$ measured with the novel $\tau$ ELISAs and the standard AlzBio3 $\tau$ and ptau assays. AD had higher total $\tau$ and ptau levels than NC and PSP. There was a gender by diagnosis interaction in $A D$ and PSP for most $\tau$ species, with lower concentrations for male compared to female patients.

Conclusions CSF $\tau$ fragment concentrations are different in PSP compared with AD despite the presence of severe $\tau$ pathology and neuronal injury in both disorders. CSF $\tau$ concentration likely reflects multiple factors in addition to the degree of neuronal injury.

\section{INTRODUCTION}

Alzheimer's disease (AD) and progressive supranuclear palsy (PSP) are two common neurodegenerative tauopathies. In both, the severity and neuroanatomic distribution of $\tau$ pathology at autopsy is correlated with disease phenomenology prior to death. ${ }^{1-3} \mathrm{AD}$ displays an equal ratio of three to four microtubule-binding repeats $(3 \mathrm{R}=4 \mathrm{R})$ and is also characterised by the accumulation of amyloid plaques in addition to $\tau$. By contrast, PSP is a relatively 'pure' tauopathy and is characterised by deposition of $4 \mathrm{R} \tau$. The prevailing hypothesis is that amyloid accumulates prior to alterations in cerebrospinal fluid (CSF) concentrations of $\tau$, which increase in parallel with neuronal damage in $\mathrm{AD}{ }^{4}$ Elevated concentrations of $\tau$ can be measured in $\mathrm{AD}$ CSF using assays that quantify central $\tau$ epitopes. Because PSP is a pure tauopathy, using tau as a biomarker may have better potential to demonstrate efficacy of $\tau$-directed therapies than studies of more pathologically heterogeneous disorders such as $\mathrm{AD}$.

PSP is less common than $\mathrm{AD}$, but is genetically and biochemically more strongly linked to $\tau .^{3}{ }^{5}$ However, CSF $\tau$ concentrations in PSP are comparable to, or lower than, age-matched controls. ${ }^{6-9}$ Similar results have been reported for human $\tau$ mutation carriers, ${ }^{10}$ and transgenic mice that express such mutations display decreasing $\tau$ concentrations in the brain interstitial fluid in parallel with deposition of insoluble $\tau$ and onset of neuronal dysfunction. ${ }^{11}$ Decreases in CSF $\tau$ in PSP and other primary tauopathies might reflect a different propensity of $4 \mathrm{R} \tau$ to aggregate, be released from cells, ${ }^{12}$ or undergo proteolytic cleavage ${ }^{13}$ as compared to equal mixtures of $3 R$ and $4 R \tau$ found in AD. ${ }^{14}$ Alternatively, standard CSF $\tau$ assays might not detect elevations of $\tau$ species that are elevated in primary tauopathies. To examine this possibility, we used novel ELISAs targeting N-terminal and middomain epitopes ${ }^{15}$ to compare CSF $\tau$ concentrations in severity-matched patients with $\mathrm{AD}$ and PSP.

\section{METHODS}

\section{Study population}

Twenty-four patients with PSP, 37 patients with $\mathrm{AD}$, and 26 normal controls (NC) were evaluated at the University of California, San Francisco Memory and Aging Center. Patients with PSP were diagnosed with possible or probable PSP according to the National Institute of Neurological Disorders and Stroke-Society for Progressive Supranuclear Palsy (NINDS-SPSP) criteria. ${ }^{16} \mathrm{~A}$ second set of patients with $\mathrm{AD}(\mathrm{n}=13)$ that were matched in age, education and gender, which were purchased from Precision Med (Solana Beach, California, USA), were also analysed due to unexpectedly high Abeta levels in the initial $\mathrm{AD}$ group. Patients with $\mathrm{AD}$ met the National Institute of Neurological and Communicative Disorders and Stroke-Alzheimer's 
Disease and Related Disorders Association (NINCDS-ADRDA) criteria for probable Alzheimer's disease. ${ }^{17} \mathrm{NC}$ reported no cognitive symptoms and had normal neurological and neuropsychological examinations. All aspects of the study were approved by the UCSF Institutional Review Board, and written informed consent was obtained from all participants.

\section{Clinical evaluations}

All individuals from UCSF received a neuropsychological assessment that included the Mini-Mental State Exam (MMSE) ${ }^{18}$; the second set of patients with $\mathrm{AD}$ were also evaluated with the MMSE. Visuospatial abilities were examined using the Benson Figure copy (Rey). ${ }^{19}$ Immediate and working memory was assessed using the forward (FDS) and backward (BDS) digit spans. ${ }^{20}$ Episodic memory was measured by the short form of the California Verbal Learning Test-2 (CVLT-2, including $30 \mathrm{~s}$ and 10 min delays), ${ }^{21}$ and 10 min delayed-recall of the Benson figure (Rey10 m). Executive function was tested using a modified Trail-making test (lines per second). ${ }^{22}$ Phonemic fluency (D words per minute), ${ }^{23}$ category fluency (animals per minute), ${ }^{23}$ and a 15 -item Boston naming task $(\mathrm{BNT})^{24}$ examined language capacities. Clinical dementia rating $(\mathrm{CDR})^{25}$ and sum of boxes $(\mathrm{CDR}-\mathrm{sb})^{12}$ scores assessed disease burden and functional abilities. The Unified Parkinson's Disease Rating Scale (UPDRS) motor quantified parkinsonism in all groups. ${ }^{26}$

\section{Lumbar punctures (LP)}

LPs were performed fasting and sitting up, in accordance with the Alzheimer's Disease Neuroimaging Initiative (ADNI) recommended procedure (http://www.adni-info.org/ADNIStudyProcedures/Lumbar Punctures.aspx), and occurred within a maximum of 5 months of the clinical assessment. Samples were collected in the morning after a $12 \mathrm{~h}$ fast, into sterile $13 \mathrm{~mL}$ polypropylene tubes. The first $2 \mathrm{~mL}$ were discarded. CSF was then transferred into $1 \mathrm{~mL}$ aliquots and put on dry ice for an hour before storing in a $-80^{\circ} \mathrm{C}$ freezer.

\section{CSF ELISAs}

INNO BIA AlzBio3 (Fujirebio/Innogenetics), a multiplex assay for $\mathrm{A} \beta_{1-42}$, $\tau$, and ptau, was performed using standard methods, including a set of three quality control (QC) standards. The novel ELISAs were performed using previously described methods. ${ }^{15}$ Different combinations of standard monoclonal antibodies to $\mathrm{N}$-terminal and central epitopes were developed to detect different overlapping regions of the $\tau$ protein. The following capture and readout antibodies combinations were used: Tau12-HT7, Tau12-BT2, BT2-HT7, HT7-Tau5, and HT7-PT181 (figure 1). Standards and samples were run in triplicate; replicate variability was $<20 \%(\mathrm{CV})$. All samples were included in the same assay run; interplate QC variability was $<10 \%(\mathrm{CV})$. The assays demonstrated $\sim 100$-fold dynamic range of quantitation, with limit of quantification (LLQ) ranging from $2 \mathrm{pg} / \mathrm{mL}$ to $10 \mathrm{pg} / \mathrm{mL} .{ }^{15} \mathrm{CSF}$ signal specificity was confirmed using a combination of immunodepletion, spike recovery and peptide competition. ${ }^{15}$ One patient with PSP was an outlier on all analyses (>2.5 SDs from the group mean) and was excluded from the analyses.

\section{Genotyping}

Genotyping of the ApoE allele (rs429358 and rs7412) and MAPT (rs1560310) H1 or H2 haplotype defining variants was carried out using a TaqMan Allelic Discrimination Assay on an ABI 7900HT Fast Real-Time PCR system (Applied Biosystems, Foster City, California, USA) according to the manufacturer's instructions, and using the same methodology as previously described. ${ }^{27}$

\section{Statistical analyses}

Data were analysed using SPSS (V.21, SPSS, Chicago) and R (http://www.r-project.org). Normality for individual variables was determined by the Shapiro-Wilk test. Depending on normality, mean values for demographic, neuropsychological and CSF analyte levels between each group were compared using a univariate analysis of variance (ANOVA) with False Discovery Rate (FDR) corrected posthoc analysis, or a Kruskal-Wallis test with a FDR corrected Mann-Whitney U test for posthoc analysis. Additionally, a linear regression model was used to examine differences in CSF biomarker values across diagnostic groups, and to investigate interactions between diagnostic group and gender, age, ApoE genotype, and $\tau$ haplotype. Although there were group differences in education levels, education did not explain a significant amount of the variance in the mean CSF concentrations (all p values $>0.443$ ) and was therefore not included in the model. Because the distribution of CSF biomarkers was skewed, inferences were performed using a resamplingbased technique that does not require (normal) distributional assumptions. Specifically, accuracy of parameter estimates was examined using a non-parametric bootstrap technique with 2500 iterations. Bootstrap (or a general linear model) posthoc testing within each diagnostic group was used to describe differences in CSF biomarkers between groups. Statistical significance was examined based on $p$ values generated by bootstrap analysis, accompanied by F-statistic values and degrees of freedom from ANOVA and FDR corrected for multiple comparisons. Lastly, a receiver operating characteristic (ROC) curve analysis using a non-parametric approach was performed to explore diagnostic accuracy of the CSF $\tau$ and ptau assays that demonstrated lower levels in patients with PSP compared to the other groups.

\section{RESULTS}

\section{Demographics and neuropsychological performance}

There were no differences in gender, age and ApoE4 and Tau Haplotype status between groups. CDR and CDR-sb scores were higher in patients with $\mathrm{AD}$ and PSP compared to $\mathrm{NC}$ $(p<0.001)$ but not different between patient groups. Disease duration (defined as the interval from disease onset to the time of CSF collection) was shorter in AD than PSP $(p=0.016)$. Patients with PSP and AD had fewer years of education than NC $(p<0.01)$. Patients with PSP were impaired relative to NC on the MMSE, CVLT 30 s, Rey, Rey10 m, FDS, BDS, and phonemic and category fluency $(\mathrm{p}<0.026)$, and had higher UPDRS scores $(p<0.001)$ (table 1). Patients with PSP never performed worse on any neuropsychological test than patients with AD. Patients with AD performed worse than NC on modified trails (MT) correct, CVLT $10 \mathrm{~min}$ delay, MMSE, Rey10 m, and BDS $(p<0.001)$. AD also had worse performance than patients with PSP on the MMSE, CVLT $30 \mathrm{~s}$ and $10 \mathrm{~min}$ delay, Rey10 m, and BDS $(p<0.007)$ but had less parkinsonism on the UPDRS $(p<0.001)$. Patients with AD performed worse than patients with NC and PSP on the BNT $(p<0.001)$.

\section{CSF analytes}

Group differences in mean CSF analyte concentrations from the novel ELISAs, and AlzBio3 ELISAs were examined using linear models. As shown in figure 2, lower CSF $\tau$ levels were identified in PSP as compared to NC with the novel $\tau$ ELISAs in several $\mathrm{N}$-terminal and mid-domain epitopes: Tau12-BT2 $(\mathrm{p}=0.018)$, BT2-HT7 $(p=0.019)$, HT7-PT181 $(p=0.05)$ and the AlzBio3 assays for total $\tau(p=0.034)$ and ptau $(p=0.028)$. Lower $A \beta$ levels were seen in PSP $(p=0.002)$ than NC. Compared to NC, 


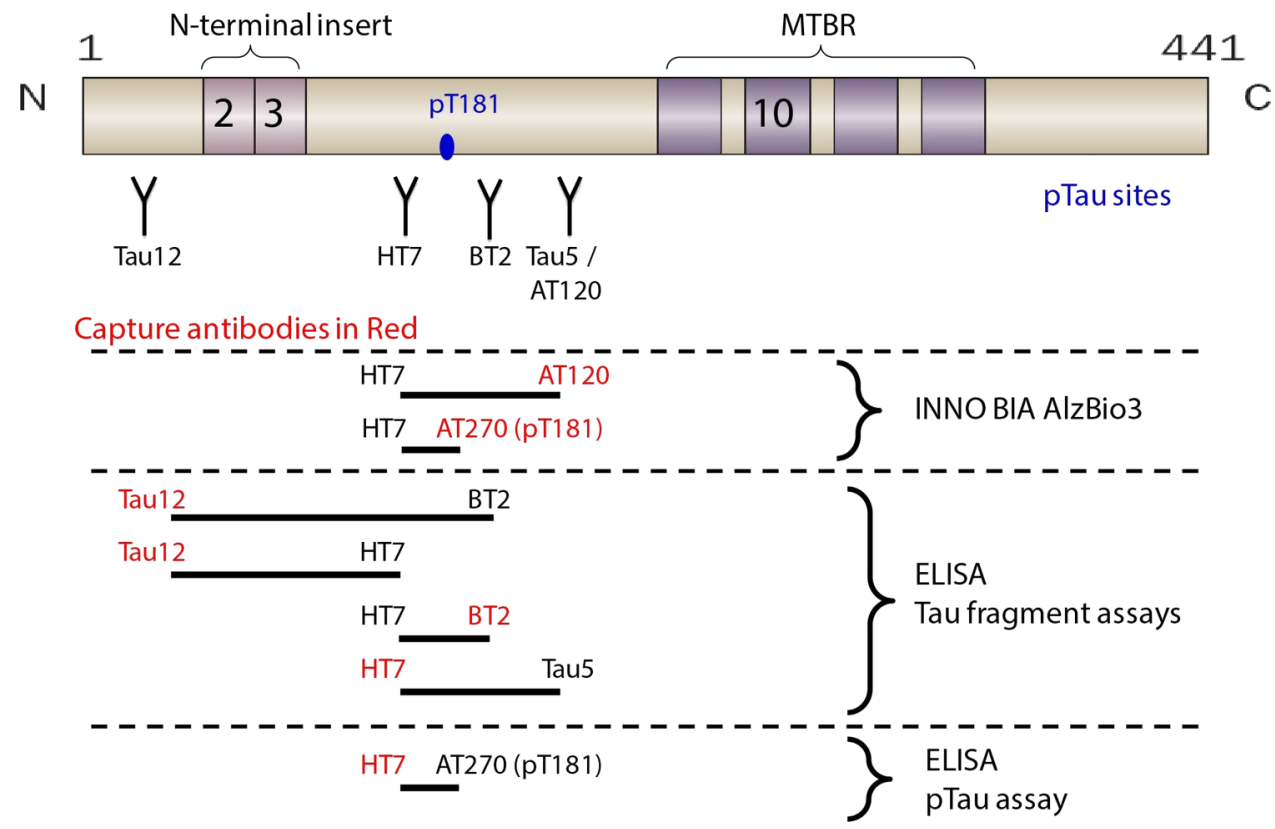

Figure 1 Tau epitopes assayed Schematic of $\tau$ protein indicating binding sites for standard monoclonal antibodies used for ELISAs from this study as well as INNOBIA AlzBio3 assays. Lines indicate relative size and location of potential fragments detectable by each assay 15 . Control ELISAs were designed to detect similar $\tau$ fragments as AlzBio3. MTBR, microtubule binding repeats. Numbers on $\tau$ schematic indicate alternatively spliced exons. ptau, phosphorylated $\tau$.

patients with $\mathrm{AD}$ displayed lower $\mathrm{A} \beta$ levels $(\mathrm{p}<0.001)$ and higher CSF $\tau$ levels in all the novel ELISAs $(p<0.013)$ and AlzBio3 total $\tau(p=0.012)$ and ptau $(p=0.01)$ assays. There were no differences between patients with AD and PSP for A $\beta$. Patients with AD also had higher CSF $\tau$ levels than patients with PSP in all the novel ELISAs $(p<0.007)$, AlzBio3 total $\tau$ $(\mathrm{p}<0.001)$ and ptau $(\mathrm{p}<0.001)$ assays (table 2$)$.

Because $A \beta$ levels were higher than expected in the initial AD group (AD1), an additional analysis in a different group of patients with $\mathrm{AD}(\mathrm{AD} 2)$ was pursued. This group demonstrated lower A $\beta$ levels than PSP $(p<0.0014)$ and NC $(p<0.0001)$. Patients with PSP had lower A $\beta$ levels than NC $(p<0.0014)$. Differences in $\tau$ levels were also analysed. The results with this $\mathrm{AD}$ group were similar to the original AD cohort (table 2).

There was an interaction between gender and CSF $\tau$ concentration in the AD1 and PSP groups, but not AD2 or NC (figure 2). Male patients with PSP had lower CSF $\tau$ levels than female patients in the N-terminal fragments Tau12-BT2 $(p=0.003)$ and Tau12-HT7 $(\mathrm{p}=0.013)$, and in the mid-domain fragments BT2-HT7 $(p=0.028)$ and HT7-Tau5 $(p=0.008)$ ELISAs. The same pattern was seen in patients with $\mathrm{AD}$; males had lower CSF $\tau$ levels than females in all the novel ELISAs $(\mathrm{p}<0.007)$, AlzBio3 total $\tau$ $(p=0.002)$ and ptau $(p=0.001)$ assays. None of the other demographic or genetic variables influenced CSF analyte concentrations.

ROC analyses were used to determine the ability of CSF $\tau$ concentration to differentiate PSP from NC and AD (table 3). Multiple CSF $\tau$ assays were able to differentiate PSP from AD (area under the curve (AUC) range between 0.794 and 0.948) with the $\mathrm{N}$-terminal fragment $\tau 12$-BT2 assay (AUC $=0.948$, $\mathrm{p}<0.001$ ) having the highest AUC value. Most CSF $\tau$ assays also differentiated PSP from NC, however AUC values were lower (AUC range between 0.654 and 0.730 ), $\mathrm{p}<0.02$.

\section{DISCUSSION}

We identified divergent patterns of CSF $\tau$ abnormalities in two common tauopathies, AD and PSP, using the standard AlzBio3
CSF $\tau$ assays used in most AD research, and a series of novel ELISAs designed to detect other CSF $\tau$ fragments. ${ }^{15}$ In patients with $\mathrm{AD}$ and PSP matched for disease severity, patients with $\mathrm{AD}$ displayed higher CSF $\tau$ and ptau levels than NC, whereas patients with PSP had lower levels than NC. In patients with PSP and one of the AD cohorts, there was an interaction with gender, such that males had lower CSF $\tau$ levels than females in most assays. There were no effects of other demographic factors or the disease-associated genotypes ApoE4 and $\tau \mathrm{H} 1$ haplotype. Although the current literature, which is mainly based on studies of $\mathrm{AD}$, suggests that elevated CSF $\tau$ concentrations reflect neuronal injury and cell death in neurodegenerative disease, ${ }^{4} 28$ our data demonstrate that this model does not hold true for the primary four repeat tauopathy, PSP. Further investigations into the mechanisms of CSF $\tau$ alterations may reveal differences in the pathophysiology of $\tau$-related neurodegeneration in $\mathrm{AD}$ and PSP. ${ }^{11} 29$

The standard AlzBio3 $\tau$ assays may detect only a subset of potential CSF $\tau$ fragments. ${ }^{15} 3031$ Therefore, we used novel CSF $\tau$ ELISAs designed to measure $\tau$ fragments containing $\mathrm{N}$-terminal and mid-domain sequences. The absolute concentrations of $\tau$ fragments measured in the novel ELISAs were higher than those measured with AlzBio3. This likely resulted from technical differences in the assays due to differences in antibodies, matrix effects or standards. Without a more quantitative measurement of $\tau$ fragment levels, such as mass spectrometry, it is impossible to directly compare these values. Nonetheless, using these assays, we found lower $\tau$ fragment levels in patients with PSP compared to controls and AD.

Unexpectedly, in the first AD cohort (AD1), CSF A 342 levels in patients with $\mathrm{AD}$ and PSP were comparable, and lower than $\mathrm{NC}$ as in one previous study, ${ }^{8}$ but different from other reports in PSP that found higher A $\beta 42$ levels. ${ }^{7} 83233$ One possible explanation for low CSF A 342 in some patients with PSP would be the presence of concurrent $\mathrm{AD}$ pathology. ${ }^{34}$ To further explore the potential confounding effects of concurrent $\mathrm{AD}$ 
Table 1 Demographic and neuropsychological variables

\begin{tabular}{|c|c|c|c|c|}
\hline Variable & NC & AD1 & AD2 & PSP \\
\hline Education & $17.8 \pm 1.6$ & $16.1 \pm 4.3^{*}$ & $15 \pm 1.15 \dagger$ & $15.8 \pm 2.3 \ddagger$ \\
\hline Gender (M/F) & $26 / 11$ & $21 / 16$ & $7 / 6$ & $11 / 12$ \\
\hline Age & $62.9 \pm 12.5$ & $64.1 \pm 9.1$ & $68.23 \pm 6.23$ & $68.0 \pm 6.7$ \\
\hline ApoE4 status (non-carrier/carrier) & $15 / 10$ & $21 / 16$ & & $16 / 3$ \\
\hline Tau haplotype (H1/H2 vs H1/H1) & $6 / 16$ & $12 / 22$ & & $2 / 18$ \\
\hline Disease duration & NA & $4.5 \pm 3.6 \S$ & & $6.3 \pm 2.6$ \\
\hline CDR-total & $0 \eta_{,}^{* *}$ & $0.7 \pm 0.7$ & & $0.4 \pm 0.5$ \\
\hline CDR-sb & $0 \|^{* *}$ & $4.9 \pm 3.0$ & & $4.4 \pm 2.5$ \\
\hline MMSE & $29.2 \pm 1.0$ & $20.4 \pm 5.9^{*}, \S$ & $28.46 \pm 2.99+\dagger$ & $26.2 \pm 3.6 \ddagger$ \\
\hline Rey & $15.5 \pm 0.7$ & $12.1 \pm 4.5^{*}$ & & $13.1 \pm 1.9 \ddagger$ \\
\hline Rey $10 \mathrm{~m}$ & $11.9 \pm 3.3$ & $4.6 \pm 4.1^{*}, \S$ & & $9.4 \pm 2.8 \ddagger$ \\
\hline FDS & $7.4 \pm 0.8$ & $4.8 \pm 1.5^{*}$ & & $5.6 \pm 1.3 \ddagger$ \\
\hline BDS & $6.3 \pm 1.0$ & $2.9 \pm 1.0^{*}, \S$ & & $3.9 \pm 1.3 \ddagger$ \\
\hline Phonemic fluency & $15.4 \pm 4.5$ & $8.2 \pm 4.4^{*}$ & & $6.8 \pm 4.0 \ddagger$ \\
\hline Category fluency & $25.0 \pm 4.1$ & $9.8 \pm 5.5^{*}$ & & $10.9 \pm 4.2 \ddagger$ \\
\hline BNT & $14.8 \pm 0.4$ & $10.9 \pm 3.8^{*}, \S$ & & $14.1 \pm 1.0$ \\
\hline Trails correct & $14.0 \pm 0$ & $8.8 \pm 5.3^{*}$ & & $12.8 \pm 2.4$ \\
\hline UPDRS-III & $0.3 \pm 0.7 \eta_{,}^{* *}$ & $9.0 \pm 12.2 \S$ & & $32.1 \pm 8.2$ \\
\hline CVLT $30 \mathrm{~s}$ & $8.4 \pm 1.1$ & $3.2 \pm 2.0^{*}, \S$ & & $6.7 \pm 1.6 \ddagger$ \\
\hline CVLT $10 \mathrm{~min}$ & $8.3 \pm 1.4$ & $2.0 \pm 2.4^{*}, \S$ & & $6.3 \pm 2.0 \ddagger$ \\
\hline
\end{tabular}

${ }^{*} A D 1<N C(p<0.05)$.

$+N C<A D 2(p<0.05)$.

$\ddagger P S P<N C(p<0.05)$.

$\S A D 1<P S P(p<0.05)$.

INC $<$ AD1 $(p<0.05)$

${ }^{* *} \mathrm{NC}<\mathrm{PSP}(\mathrm{p}<0.05)$.

$++P S P<A D 2(p<0.05)$

AD1, Alzheimer's disease in the original cohort; AD2, Alzheimer's disease in the second cohort; ApoE4 status, ApoE4 non-carrier vs carrier; BDS, Digit Span Backward; BNT, Boston naming task (out of 15); Categorical Fluency, number of animals in 1 min; CDR, Clinical Dementia Rating; CDR- sb, Clinical Dementia Rating sum of boxes; Disease duration, number of years from the onset of the disease to the date of CSF collection; CVLT $30 \mathrm{~s}$ and 10 min, number of correct items remembered on the California verbal learning task after a $30 \mathrm{~s}$ or 10 min delay; FDS, Digit Span Forward; MMSE, Mini-Mental State Examination; NC, normal control; Phonemic Fluency, number of correct words beginning with D in 1 min; PSP, progressive supranuclear palsy; Rey and Rey10, Benson Figure copy test immediate recall and 10 min delayed recall; Trails Correct, Modified Trail-making test number of correct items; UPDRS-III, Unified Parkinson's Disease Rating Scale-part III motor scale.

pathology we compared PSP cases with low A $\beta 42$ (below the mean) with those with higher $A \beta 42$ (mean or higher) and found that patients with PSP with low CSF A $\beta 42$ had even lower total $\tau$ levels and $\tau$ fragment levels than patients with PSP with high CSF A $\beta 42$ levels $(p<0.034)$. This suggests that even in combined PSP and AD, CSF $\tau$ levels are lower than NCs, however, further analyses in autopsy-confirmed cases will be necessary to confirm this hypothesis.

We also found lower CSF total $\tau$ and ptau levels using the standard AlzBio3 assays in PSP compared to AD and controls. Although several previous studies have found PSP CSF total $\tau$ concentrations comparable to controls, ${ }^{7} 832$ the lack of observed differences in those studies may have been confounded by $\tau$ levels being near the limit of detection; a more recent study $^{6}$ demonstrated lower ptau in PSP compared to controls, similar to our results.

There was an effect of gender on CSF $\tau$ levels measured in many of the $\tau$ assays in AD1 and PSP, but not in NC. AD1 and male patients with PSP had lower CSF $\tau$ than their female counterparts (figure 2). This suggests that our ability to detect CSF $\tau$ concentration differences between PSP and NC could also reflect differences in the gender composition of both groups as compared to previous studies. A previous study demonstrated that normal elder female ApoE4 carriers have increased CSF $\tau$ levels relative to non-carriers, similar to our results in $\mathrm{AD} .{ }^{35}$ Another study found patients with AD who are $\tau \mathrm{H} 1 / \mathrm{H} 1$ haplotype carriers have lower CSF $\tau$ levels as compared to $\mathrm{H} 1 / \mathrm{H} 2$ carriers. ${ }^{36}$ We did not observe either effect, but differences in sample size, age and gender distribution may have influenced our findings since the gender effect was not present in the AD2 group.

A limitation of this study is the difficulty in matching patients with $\mathrm{AD}$ and PSP for disease severity given their disparate clinical presentations. We matched patient groups using the CDR-sb which is commonly used to match $\mathrm{AD}$ with non-AD neurodegenerative syndromes. $^{33} 37$ While it is possible the relative disease severity differed between patient groups, the different directions of CSF $\tau$ alteration relative to NC argue that such differences would not influence our results, and there were no correlations between disease severity and CSF $\tau$ levels in either patient group. A second potential limitation of this work is that we relied on clinical and not neuropathological diagnoses that could quantify the exact degree of underlying $\tau$ pathology. Given the disparate clinical presentations and excellent clinicpathological diagnostic accuracy of the PSP criteria we used, ${ }^{16}$ it is unlikely that misdiagnosis confounded these results; however, it is possible that our results might be influenced by the presence of concurrent $\mathrm{AD}$ pathology, which sometimes occurs in PSP. ${ }^{34}$ A third potential limitation of this study is that the novel ELISAs could not distinguish between $3 \mathrm{R}$ and $4 \mathrm{R} \tau$ isoforms. A previous study ${ }^{38}$ found lower $4 \mathrm{R} \tau$ in CSF from patients with $\mathrm{AD}$ and PSP, suggesting that the difference we observed could potentially be explained by a selective alteration in $3 \mathrm{R} \tau$ levels. Future studies will examine this possibility.

Our findings suggest that the relationship between CSF $\tau$ and neurodegenerative disease is a complex process potentially 

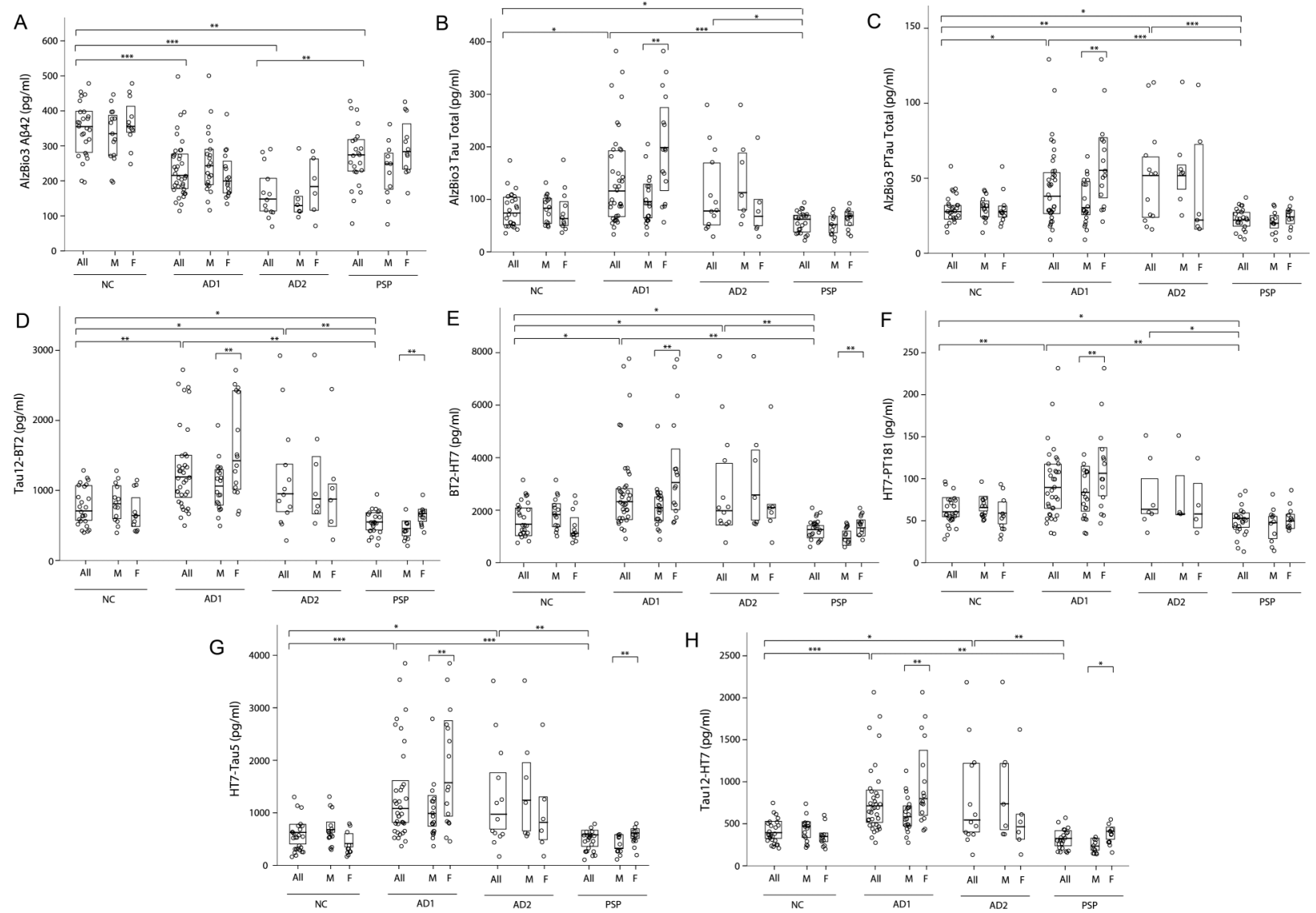

Figure 2 Diagnostic group differences in CSF analytes stratified by gender CSF concentrations of $\beta$-amyloid peptide1-42 (Aß42) (A), Alzbio3 total tau (Tau) (B), AlzBio3 phosphorylated tau (PTau) (C), Tau12-BT2 tau fragment (D), BT2-HT7 $\tau$ fragment (E), HT7-PTau181 tau fragment (F), HT7-Tau5 $\tau$ fragment $(\mathrm{G})$, and Tau12-HT7 $\tau$ fragment $(\mathrm{H})$, in normal controls $(\mathrm{NC})$ and patients with Alzheimer's disease (AD) and progressive supranuclear palsy (PSP); All $=$ total number of subjects in the group; $M$, male subjects; $F$, female subjects. ${ }^{*} p<0.05 .{ }^{* \star} p<0.01 .{ }^{* \star *} p<0.001$.

reflecting multiple factors related to the underlying cause of the tauopathy. Therefore, viewing $\tau$ only as an indicator of neuronal damage may not be accurate in generating models of $\tau$-related pathogenesis. Possible explanations for the different effects of disease on CSF $\tau$ concentrations measured using ELISA-based techniques in different tauopathies include (1) Altered CSF $\tau$ fragment conformations may lead to differential affinity for the same monoclonal antibody. (2) Differences in CSF $\tau$ fragments produced in each disease may lead to different observed patterns in CSF. ${ }^{13}{ }^{39}$ We found the greatest differences between PSP and NC using the N-terminal fragment ELISAs Tau12, BT2 and HT7; selective alterations in N-terminal $\tau$ fragments in PSP $^{13} 40$

Table 2 Mean CSF analyte concentrations

\begin{tabular}{|c|c|c|c|c|}
\hline Variable & NC & AD1 & AD2 & PSP \\
\hline AlzBio3 $A \beta_{42}(p g / m L)$ & $348.1 \pm 75.7$ & $237.5 \pm 82.7^{*}$ & $167.06 \pm 73.49 \dagger, \ddagger$ & $269.0 \pm 87.0 \S$ \\
\hline AlzBio3 total $\tau(\mathrm{pg} / \mathrm{mL})$ & $79.0 \pm 33.19$ & $138.4 \pm 88.6$ & $111.09 \pm 78.17$ & $55.9 \pm 20.1 \S_{,}{ }^{* *}, t \dagger$ \\
\hline AlzBio3 ptau (pg/mL) & 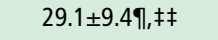 & $44.7 \pm 25.7$ & $50.47 \pm 33.15$ & $22.1 \pm 7.4 \S_{,}{ }^{* *}, \dagger \dagger$ \\
\hline Tau12-BT2 (pg/mL) & $773.3 \pm 275.81, \neq \ddagger$ & $1312.4 \pm 591.5$ & $1180.03 \pm 774.95$ & $549.3 \pm 173.9 \S_{,}{ }^{* *}, \dagger \dagger$ \\
\hline BT2-HT7 (pg/mL) & $1587.2 \pm 652.99$, $\neq \ddagger$ & $2719.4 \pm 1630.1$ & $2786.06 \pm 2079.67$ & $1205.6 \pm 390.2 \S,{ }^{* *}, \dagger \dagger$ \\
\hline HT7-Tau5 (pg/mL) & $639.7 \pm 300.19, \neq \ddagger$ & $1442.1 \pm 909.6$ & $1353.13 \pm 989.99$ & $546.2 \pm 186.5^{* *}, \mathrm{t \dagger}$ \\
\hline HT7-PTau181 (pg/mL) & $64.0 \pm 17.4 \eta$,キ‡ & $96.4 \pm 42.1$ & $82.06 \pm 42.49$ & $51.7 \pm 17.5 \S_{,}^{* *},+\dagger$ \\
\hline Tau12-HT7 (pg/mL) & $409.9 \pm 141.99, \neq \ddagger$ & $788.2 \pm 414.0$ & $789.08 \pm 603.01$ & $321.5 \pm 117.7^{* *},+\dagger$ \\
\hline
\end{tabular}


Table 3 Receiver operating characteristic (ROC) curve analysis

\begin{tabular}{lll}
\hline ROC analysis & AUC & Asymp. significance, $p$ Value \\
\hline PSP vs NC & & \\
AlzBio3 total $\tau$ & 0.702 & 0.015 \\
AlzBio3 ptau & 0.716 & 0.010 \\
Tau12-BT2 & 0.730 & 0.006 \\
BT2-HT7 & 0.654 & 0.065 \\
HT7-PTau181 & 0.694 & 0.020 \\
PSP vs AD1 & & \\
AlzBio3 total $\tau$ & 0.846 & $<0.001$ \\
AlzBio3 ptau & 0.794 & $<0.001$ \\
Tau12-BT2 & 0.948 & $<0.001$ \\
BT2-HT7 & 0.904 & $<0.001$ \\
HT7-PTau181 & 0.861 & $<0.001$ \\
PSP vs NC and AD1 & & \\
AlzBio3 total $\tau$ & 0.787 & $<0.001$ \\
AlzBio3 ptau & 0.762 & $<0.001$ \\
Tau12-BT2 & 0.859 & $<0.001$ \\
BT2-HT7 & 0.801 & $<0.001$ \\
HT7-PTau181 & 0.790 & $<0.001$ \\
\hline
\end{tabular}

AD1, Alzheimer's disease in the original cohort; Asymp, asymptotic; AUC, area under the curve; NC, normal control; PSP: progressive supranuclear palsy.

might, therefore, partially explain these results. (3) Tau isoform ratios differ in PSP and AD, with predominantly 4R $\tau$ in PSP, and equal $3 R$ to $4 R$ ratios in $A D$. A recent study found that $4 R$ $\tau$ is not released from cells as readily as $3 R \tau{ }^{12}$ Since $4 R \tau$ accumulates in PSP, more $\tau$ may be sequestered in dying cells than in $\mathrm{AD}$, leading to lower extracellular concentrations reflected in CSF measurements.

A variety of new $\tau$-directed therapies are entering human clinical trials for AD and other tauopathies. ${ }^{14} 39$ These drugs were developed in transgenic mouse models that overexpress mutant forms of $4 \mathrm{R} \tau$, which produces human clinical disease similar to frontotemporal degeneration or PSP, but not AD. The different associations between disease and CSF $\tau$ concentration observed in this study suggest that alterations in CSF $\tau$ in clinical trials of $\tau$-directed therapies may require different interpretations in $\mathrm{AD}$ and PSP, and that results in PSP may be more analogous to treatment effects observed in preclinical models.

Contributors All authors have made substantial contributions to conception and design, acquisition of data or analysis and interpretation of data; drafting of the article or revising it critically for important intellectual content, and provided final approval of the version to be published.

Funding This study was supported by Bristol-Myers Squibb, Tau Research Consortium, and National Institutes of Health grants R01AG038791 and P01 AG19724.

Competing interests $S S, M A, H S, J M, V G$, and $T H$ are employees of Bristol-Myers Squibb (BMS). MA and HS are BMS shareholders. FB is an employee of Kyowa Hakko Kirin Pharma. JHK receives royalties from Pearson for the publication of commercially available neuropsychological tests. He receives research support from the NIH (2R01AG022983, 1R01AG032289, HHSN271200623661C). GDR receives research support from NIH/NIA (K23-AG031861), Alzheimer's Association, Tau Consortium, Hellman Family Foundation, John Douglas French Alzheimer's Foundation, and Avid Radiopharmaceuticals/Eli Lilly; he has served as a consultant for Eli Lily and received a speaking honorarium from GE Healthcare. BLM serves on a scientific advisory board for the Alzheimer's Disease Clinical Study; serves as an Editor for Neurocase and as an Associate Editor of ADAD; receives royalties from the publication of Behavioural Neurology of Dementia (Cambridge, 2009), Handbook of Neurology (Elsevier, 2009), and The Human Frontal Lobes (Guilford, 2008); serves as a consultant for Lundbeck Inc., Elan Corporation, and Allon Therapeutics, Inc.; serves on speakers' bureaus for Novartis and Pfizer Inc.; and receives research support from Novartis and the NIH (NIA P50 AG23501 (PI) and NIA P01 AG19724 (PI)) and the State of California Alzheimer's Center. ALB received personal compensation from Acetylon, Archer, Ipierian and Neurophage for consulting and received research support from Genentech, BMS, TauRx for conducting clinical trials. He is funded by NIH grants R01AG038791 (PI), R01AG031278 (PI), the Alzheimer's Drug Discovery Foundation, CBD Solutions and the Tau Research Consortium.

Ethics approval UCSF Institutional Review Board.

Provenance and peer review Not commissioned; externally peer reviewed.

\section{REFERENCES}

1 Braak H, Braak E. Neuropathological stageing of Alzheimer-related changes. Acta Neuropathol 1991;82:239-59.

2 Schofield EC, Hodges JR, Bak TH, et al. The relationship between clinical and pathological variables in Richardson's syndrome. J Neurol 2012;259:482-90.

3 Williams DR, Lees AJ. Progressive supranuclear palsy: clinicopathological concepts and diagnostic challenges. Lancet Neurol 2009;8:270-9.

4 Jack CR Jr, Knopman DS, Jagust WJ, et al. Hypothetical model of dynamic biomarkers of the Alzheimer's pathological cascade. Lancet Neurol 2010;9:119-28.

5 Boxer AL, Gold M, Huey E, et al. The advantages of frontotemporal degeneration drug development (part 2 of frontotemporal degeneration: the next therapeutic frontier). Alzheimers Dement 2013;9:189-98.

6 Hall S, Ohrfelt A, Constantinescu R, et al. Accuracy of a panel of 5 cerebrospinal fluid biomarkers in the differential diagnosis of patients with dementia and/or parkinsonian disorders. Arch Neurol 2012;69:1445-52.

7 Urakami K, Wada K, Arai H, et al. Diagnostic significance of tau protein in cerebrospinal fluid from patients with corticobasal degeneration or progressive supranuclear palsy. J Neurol Sci 2001;183:95-8.

8 Noguchi M, Yoshita M, Matsumoto $Y$, et al. Decreased beta-amyloid peptide42 in cerebrospinal fluid of patients with progressive supranuclear palsy and corticobasal degeneration. J Neurol Sci 2005;237:61-5.

9 Frank RA, Galasko D, Hampel H, et al. Biological markers for therapeutic trials in Alzheimer's disease. Proceedings of the biological markers working group; NIA initiative on neuroimaging in Alzheimer's disease. Neurobiol Aging 2003:24:521-36

10 Rosso SM, van Herpen E, Pijnenburg YA, et al. Total tau and phosphorylated tau 181 levels in the cerebrospinal fluid of patients with frontotemporal dementia due to P301L and G272V tau mutations. Arch Neurol 2003;60:1209-13.

11 Yamada K, Cirrito JR, Stewart FR, et al. In vivo microdialysis reveals age-dependent decrease of brain interstitial fluid tau levels in P301S human tau transgenic mice. J Neurosci 2011;31:13110-17.

12 Karch CM, Jeng AT, Goate AM. Extracellular Tau levels are influenced by variability in Tau that is associated with tauopathies. J Biol Chem 2012;287:42751-62.

13 Arai $\mathrm{T}$, Ikeda $\mathrm{K}$, Akiyama $\mathrm{H}$, et al. Identification of amino-terminally cleaved tau fragments that distinguish progressive supranuclear palsy from corticobasal degeneration. Ann Neurol 2004;55:72-9.

14 Brunden KR, Trojanowski JQ, Lee VM. Advances in tau-focused drug discovery for Alzheimer's disease and related tauopathies. Nat Rev Drug Discov 2009;8:783-93.

15 Meredith JE Jr, Sankaranarayanan S, Guss V, et al. Characterization of Novel CSF Tau and ptau Biomarkers for Alzheimer's Disease. PLoS One 2013;8:e76523.

16 Litvan I, Agid Y, Calne D, et al. Clinical research criteria for the diagnosis of progressive supranuclear palsy (Steele-Richardson-Olszewski syndrome): report of the NINDS-SPSP international workshop. Neurology 1996;47:1-9

17 McKhann G, Drachman D, Folstein M, et al. Clinical diagnosis of Alzheimer's disease: report of the NINCDS-ADRDA Work Group under the auspices of Department of Health and Human Services Task Force on Alzheimer's Disease. Neurology 1984;34:939-44.

18 Folstein MF, Folstein SE, McHugh PR. "Mini-mental state". A practical method for grading the cognitive state of patients for the clinician. J Psychiatr Res 1975;12:189-98.

19 Possin KL, Laluz VR, Alcantar OZ, et al. Distinct neuroanatomical substrates and cognitive mechanisms of figure copy performance in Alzheimer's disease and behavioral variant frontotemporal dementia. Neuropsychologia 2011:49:43-8.

20 Wechsler D. Wechsler Adult Intelligence Scale. 3rd edn. San Antonio, TX: The Psychological Corporation, 1997.

21 Delis DC, Kramer JH, Kaplan E, et al. California Verbal Learning Test, Second Eed. San Antonio, TX: The Psychological Corporation, 2000.

22 Kramer JH, Jurik J, Sha SJ, et al. Distinctive neuropsychological patterns in frontotemporal dementia, semantic dementia, and Alzheimer disease. Cogn Behav Neurol 2003;16:211-18.

23 Delis DC, Kaplan EB, Kramer JH. The Delis-Kaplan executive function system. San Antonio, TX: The Psychological Corporation, 2001.

24 Kaplan E, Goodglass H, Weintraub S. Boston Naming Test. Philadelphia, PA: Lea \& Febiger, 1983.

25 Morris JC. The Clinical Dementia Rating (CDR): current version and scoring rules. Neurology 1993;43:2412-14.

26 Fahn S, Elton RL; Committee motUD. Unified Parkinson's Disease rating scale. In: Fahn S, Marsden CD, Calne D, Goldstein M, eds. Recent Developments in 


\section{General neurology}

Parkinson's Disease. Florham Park, NJ: Macmillan Health-care Information, 1987:153-63.

27 Coppola G, Chinnathambi S, Lee JJ, et al. Evidence for a role of the rare p.A152T variant in MAPT in increasing the risk for FTD-spectrum and Alzheimer's diseases. Hum Mol Genet 2012;21:3500-12

28 Zetterberg $\mathrm{H}$, Hietala MA, Jonsson $\mathrm{M}$, et al. Neurochemical aftermath of amateur boxing. Arch Neurol 2006;63:1277-80.

29 Maia LF, Kaeser SA, Reichwald J, et al. Changes in amyloid-beta and Tau in the cerebrospinal fluid of transgenic mice overexpressing amyloid precursor protein. Sci Trans/ Med 2013;5:194re192.

30 Hanisch $\mathrm{K}$, Soininen $\mathrm{H}$, Alafuzoff I, et al. Analysis of human tau in cerebrospinal fluid. J Proteome Res 2010;9:1476-82.

31 Ishiguro $\mathrm{K}$, Ohno $\mathrm{H}$, Arai $\mathrm{H}$, et al. Phosphorylated tau in human cerebrospinal fluid is a diagnostic marker for Alzheimer's disease. Neurosci Lett 1999;270:91-4.

32 Constantinescu $\mathrm{R}$, Zetterberg $\mathrm{H}$, Holmberg $\mathrm{B}$, et al. Levels of brain related proteins in cerebrospinal fluid: an aid in the differential diagnosis of parkinsonian disorders. Parkinsonism Relat Disord 2009:15:205-12.

33 Scherling CS, Hall T, Berisha F, et al. CSF neurofilament concentration reflects disease severity in frontotemporal degeneration. Ann Neurol 2014;75:116-26.
34 Gearing M, Olson DA, Watts RL, et al. Progressive supranuclear palsy: neuropathologic and clinical heterogeneity. Neurology 1994;44:1015-24

35 Damoiseaux JS, Seeley WW, Zhou J, et al. Gender modulates the APOE epsilon4 effect in healthy older adults: convergent evidence from functional brain connectivity and spinal fluid tau levels. J Neurosci 2012;32:8254-62.

36 Johansson A, Zetterberg $\mathrm{H}$, Hakansson A, et al. TAU haplotype and the Saitohin Q7R gene polymorphism do not influence CSF Tau in Alzheimer's disease and are not associated with frontotemporal dementia or Parkinson's disease. Neurodegener Dis 2005:2:28-35.

37 Liu W, Miller BL, Kramer JH, et al. Behavioral disorders in the frontal and temporal variants of frontotemporal dementia. Neurology 2004;62:742-8.

38 Luk C, Compta Y, Magdalinou N, et al. Development and assessment of sensitive immuno-PCR assays for the quantification of cerebrospinal fluid three- and four-repeat tau isoforms in tauopathies. J Neurochem 2012;123:396-405.

39 Yanamandra K, Kfoury N, Jiang $\mathrm{H}$, et al. Anti-tau antibodies that block tau aggregate seeding in vitro markedly decrease pathology and improve cognition in vivo. Neuron 2013:80:402-14.

40 Borroni B, Gardoni F, Parnetti L, et al. Pattern of Tau forms in CSF is altered in progressive supranuclear palsy. Neurobiol Aging 2009;30:34-40. 